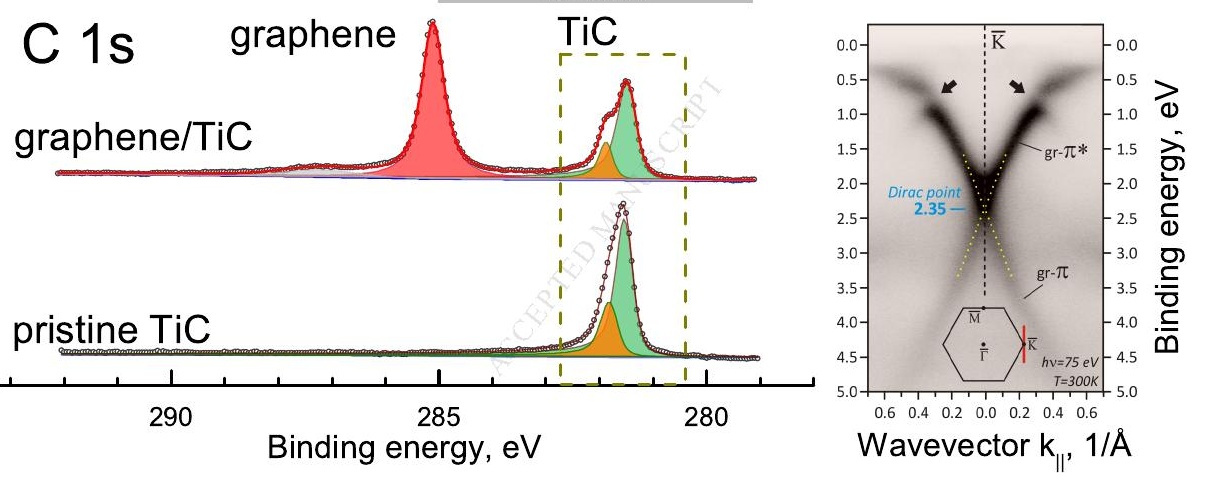




\title{
Native and Graphene-coated Flat and Stepped Surfaces of TiC
}

Elmar Yu. Kataev ${ }^{1}$, Dmitry Yu. Usachov ${ }^{2}$, Alexander S. Frolov ${ }^{1}$, Alexei A. Rulev ${ }^{1}$, Andrey A. Volykhov ${ }^{1}$, Anna Ya. Kozmenkova ${ }^{1}$, Maxim Krivenkov ${ }^{3}$, Dmitry Marchenko ${ }^{3}$, Andrei Varykhalov $^{3}$, Mikhail V. Kuznetsov ${ }^{4}$, Denis Vyalikh ${ }^{5,6}$, Lada V. Yashina ${ }^{{ }^{*}}$

1 Lomonosov Moscow State University, Leninskie gory, 119991 Moscow, Russia

2 St. Petersburg State University, 7/9 Universitetskaya nab., St. Petersburg, 199034, Russia

3 Helmholtz-Zentrum Berlin für Materialien und Energie, Albert-Einstein-Str. 15, 12489

Berlin, Germany

4 Institute of Solid State Chemistry of the Ural Branch of the Russian Academy of Sciences, Pervomayskaya Str. 91, 620990 Ekaterinburg, Russia

5 IKERBASQUE, Basque Foundation for Science, 48011 Bilbao, Spain

6 Donostia International Physics Center (DIPC), Departamento de Fisica de Materiales and CFM-MPC UPV/EHU, 20080 San Sebastian, Spain

\begin{abstract}
Titanium carbide attracts growing interest as a substrate for graphene growth and as a component of the composite carbon materials for supercapacitors, an electrode material for metal-air batteries. For all these applications, the surface chemistry of titanium carbide is highly relevant and being, however, insufficiently explored especially at atomic level is a subject of our studies. Applying X-ray photoelectron spectroscopy (XPS) to clean (111) and (755) surfaces of $\mathrm{TiC}$, we were able to obtain the detailed spectroscopic pattern containing information on the plasmon structure, shake up satellite, the peak asymmetry and, finally, surface core level shift (SCLS) in C 1s spectra. The latter is essential for further precise studies of chemical reactions. Later on, we studied interface between TiC (111) and (755) and graphene and found the SCLS variation due to strong chemical interaction between graphene and substrate. The latter is also reflected in the peculiar band structure of graphene probed by angle-resolved photoelectron spectroscopy (ARPES). Based on LEED data the structure is close to $(7 \sqrt{3} \times 7 \sqrt{3}) \mathrm{R} 30^{\circ}$, with graphene being slightly corrugated. We found that similarly to the graphene on metals, the chemical interaction between graphene and $\mathrm{TiC}$ can be weakened by means of intercalation of oxygen atoms underneath of graphene.
\end{abstract}

\footnotetext{
* Corresponding author. E-mail: yashina@inorg.chem.msu.ru (Lada Yashina)
} 


\section{Introduction}

Titanium carbide (TiC) is an iconic example of the so-called metallic "interstitial compounds". The covalent radius of the carbon atom is almost twice less than that of titanium, and carbon atoms occupy octahedral holes in the $f c c$ metal lattice, forming a rock salt structure. Upon carbon insertion, the metal-metal distances in $\mathrm{TiC}$ become slightly higher, by $0.1 \AA$, and comprise $3.05 \AA$ [1]. The Ti-C bond is covalent with a notable contribution of ionicity due to a charge transfer from titanium to carbon. Such a mixed bonding results in a unique combination of material properties, which are rather different from those of metallic titanium, such as high melting point of about $3100^{\circ} \mathrm{C}$ against $1670^{\circ} \mathrm{C}$ for Ti, high hardness of $2900-3200 \mathrm{~kg} / \mathrm{mm}^{2}$, i.e. 30 times higher than that of Ti. Besides, metallic conductivity is preserved to a certain degree due to overlapping of $d$ orbitals of the adjacent Ti atoms. Namely, the electrical resistivity of $\mathrm{TiC}$ is $68 \mu \Omega \mathrm{cm}$ against $43 \mu \Omega \mathrm{cm}$ for metallic Ti.

In addition to the physical properties discussed above, the incorporation of carbon atoms into titanium lattice and their ordering give rise to drastic changes in chemical reactivity. Namely, moderate chemical reactivity of $\mathrm{TiC}$ in comparison to that of metallic titanium leads to much better catalytic activity similar to that of noble metals in such processes as transformation of hydrocarbons and desulfurization reactions [2]. Together with some other carbides, $\mathrm{TiC}$ is an attractive substrate for graphene [3-5] or carbon nanoribbons [6] if vicinal surfaces are used. Currently titanium carbides are widely studied as a component of composite carbon materials for supercapacitors [7, 8] and as a promising electrode material for Li-air batteries $[9,10]$.

The surface chemistry of TiC plays an essential role in all cases mentioned above, and X-ray photoelectron spectroscopy (XPS) appears to be the most powerful tool to probe it. The spectral pattern of the Ti $2 \mathrm{p}$ and $\mathrm{C} 1$ s core levels for clean surface should be investigated in detail and properly quantified. This would allow to avoid misinterpretation and, finally, to precisely evaluate the surface reactivity of TiC. Titanium is a light $3 d$ element, therefore several spectral features are expected for its $2 \mathrm{p}$ peak in metallic Ti-containing compounds, such as shake-up satellites, developed plasmon structures, peak asymmetry, and the surface core level shift (SCLS). For the spectra related to single crystals, photoelectron diffraction phenomena should be also taken into account. Consistent interpretation of the $\mathrm{TiC}$ photoemission spectra still remains a challenge due to the complexity of their quantification and peak shape analysis, especially for high resolution spectra. 
Fortunately, titanium carbide is a well-studied material with respect to its chemical bonding and surface structure $[1,11,12]$; such data provide a platform for the XP-spectra interpretation. For TiC possessing a formal $d^{0}$ configuration, in contrast to TiN with a formal $d^{1}$ configuration and high density of states at the Fermi level, such spectral features as shakeup satellites, dominating for $\operatorname{TiN}[13,14]$, and peak asymmetry are questionable, it is anticipated that they should be much less pronounced [15]. It should be noted that such formal presentation is far from reality due to high contribution of covalent bond. Certain information on plasmon structure is provided in the earlier EELS studies [16]. The SCLS of about $-0.3 \mathrm{eV}$ was found in the $\mathrm{C} 1 \mathrm{~s}$ spectra of many metallic carbides both for the (100) and the (111) surfaces [17, 18], however, the information on TiC (111) is still missing. Clean TiC surfaces are known to be rather unstable towards oxidation and sensitive to the base pressure level in the vacuum chamber, and oxidation-related peaks overlap with the shake-up satellites as well as the surface plasmon spectral features.

Graphene grown on singular and different vicinal TiC surfaces has been studied earlier by LEED and STM to uncover the atomic structure of the system [3-5]. Carbon nanoribbons can be grown on the vicinal surfaces like (755) when the surface coverage is below $100 \%$ [6]. However, the electronic properties of these systems have not been deeply explored. Moreover, even less is known on the interaction between graphene and its substrate.

In the present paper, we focus on the photoemission study of clean and graphene covered $\mathrm{TiC}$ (111) and (755) surfaces. First, we consider contributions of different effects like shake-up satellites, peak asymmetry, plasmons, and, finally, the surface core level shift (SCLS). Second, for the graphene-covered surface we study the structure, chemical bonding, and electronic properties. We found the SCLS variation to be caused by graphene growth, which evidences strong interaction between graphene and TiC due to partial hybridization of the $\mathrm{C} 2 p$ and Ti $3 d$ orbitals. This interaction is clearly reflected in the band structure comprehensively evaluated by ARPES. Moreover, the graphene-substrate coupling is strongly supported by graphene substrate decoupling upon oxygen intercalation. Third, we observed that covered by graphene highly reactive titanium carbide surfaces become essentially more robust against oxidation that makes them further interesting for industrial applications.

\section{Results and Discussion}

2.1. Clean $\mathrm{TiC}(111)$ and (755) surfaces 


\subsubsection{Surface structure and chemical bonding}

$\mathrm{TiC}(111)$ is a polar surface that can be either $\mathrm{C}$ or Ti- terminated; the latter situation is commonly observed after surface preparation in UHV by heat treatment [19]. The TiC (755) surface is a vicinal plane that deviates from the (111) plane by about $9^{\circ}$, i.e. it has a stepped structure with (111) terraces that are approximately $1.3 \mathrm{~nm}$ wide. The corresponding atomic model is shown in Fig. 1a. The LEED pattern for the (111) surface displayed in Fig. 1b corresponds to a hexagonal (1x1) atomic structure. For the $\mathrm{TiC}(755)$ surface the presence of atomic steps causes splitting of spots and appearance of vertical stripes [6] (Fig. 1c). A simulated LEED pattern shown in Fig. 1d is in a good accordance with the experimental observations.

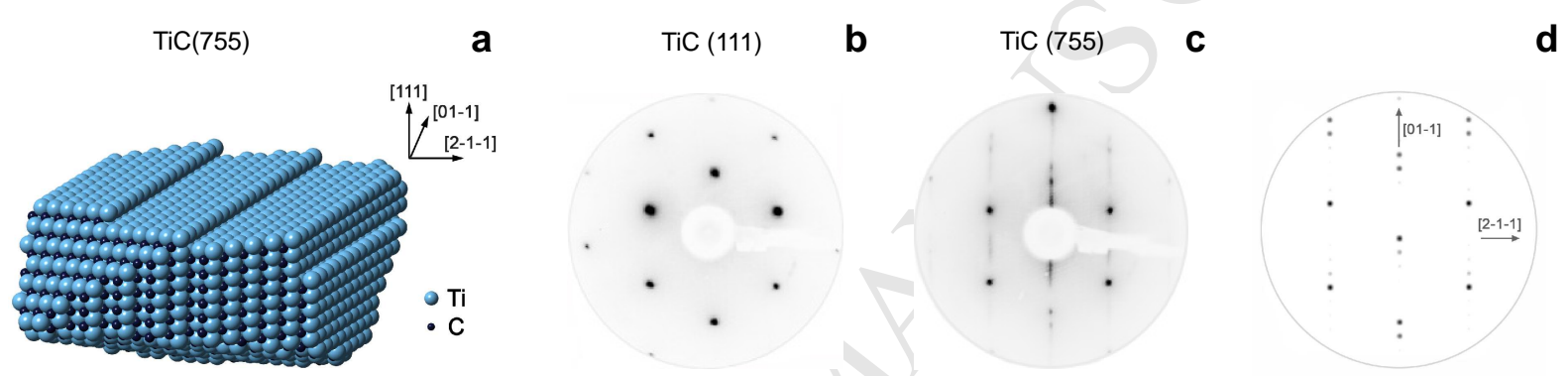

Figure 1. Atomic model for the TiC (755) surface (a), LEED patterns for TiC (111) (b), TiC (755) (c) obtained at an electron energy of $125 \mathrm{eV}$; simulated pattern for the (755) surface (d).

Figures $2 \mathrm{~b}$ and $2 \mathrm{c}$ display the near-edge X-ray absorption (NEXAFS) spectra for the $\mathrm{C}-K$ and Ti- $L$ core levels, correspondingly. The photon absorption is accompanied by transfer of core shell electrons to unoccupied valence band states above the Fermi level. Therefore, the absorption spectra can be interpreted on the basis of the corresponding molecular orbital or band-structure calculations above the Fermi level in the excited state. Generally, strict theoretical description of X-ray absorption process in solid is not straightforward since it is difficult to describe the excited system with the core hole adequately within the standard density functional theory (DFT). One can take into account that, first, for titanium carbide the bonding is dominated by a strong directional covalent Ti-C bond [11, 20]. Second, transfer of electrons from $\mathrm{Ti}$ to $\mathrm{C}$ takes place, thus implying a certain degree of ionic bonding. Third, as it follows from Fig. 2a the DOS does not vanish at the Fermi energy, therefore giving the compounds a metallic character. The dominating covalent bonding allows one to interpret the absorption spectra in terms of molecular orbitals in the first approximation as shown in 
Fig. 2d [21]. According to this simplified approach, the low energy feature in the C- $K$ spectrum in Fig. 2b corresponds to a resonant transition from the $\mathrm{C}-K$ core level to the unoccupied $\pi^{*} 2 t_{2 g}$ molecular orbital composed by the Ti $t 2 g$ and C $2 p$ atomic orbitals; other features and their energies are summarized in Table 1 . This strongly simplified molecular orbital scheme is useful to give a hint on hybrid orbital shape and generally agrees well with the partial densities of states obtained by our DFT modeling in the initial state approximation of bulk TiC as presented in Fig. 2a. The calculated DOS above the Fermi level, in turn, describes well $\mathrm{C}-K$ absorption spectrum and, below the Fermi level, reasonably fits the valence band shape presented in the left panel of Fig. 2b. Quantitative comparison of the corresponding energies based on our DFT modelling is given in Table 1. Minor discrepancies in energies are probably attributed to the final state contribution.
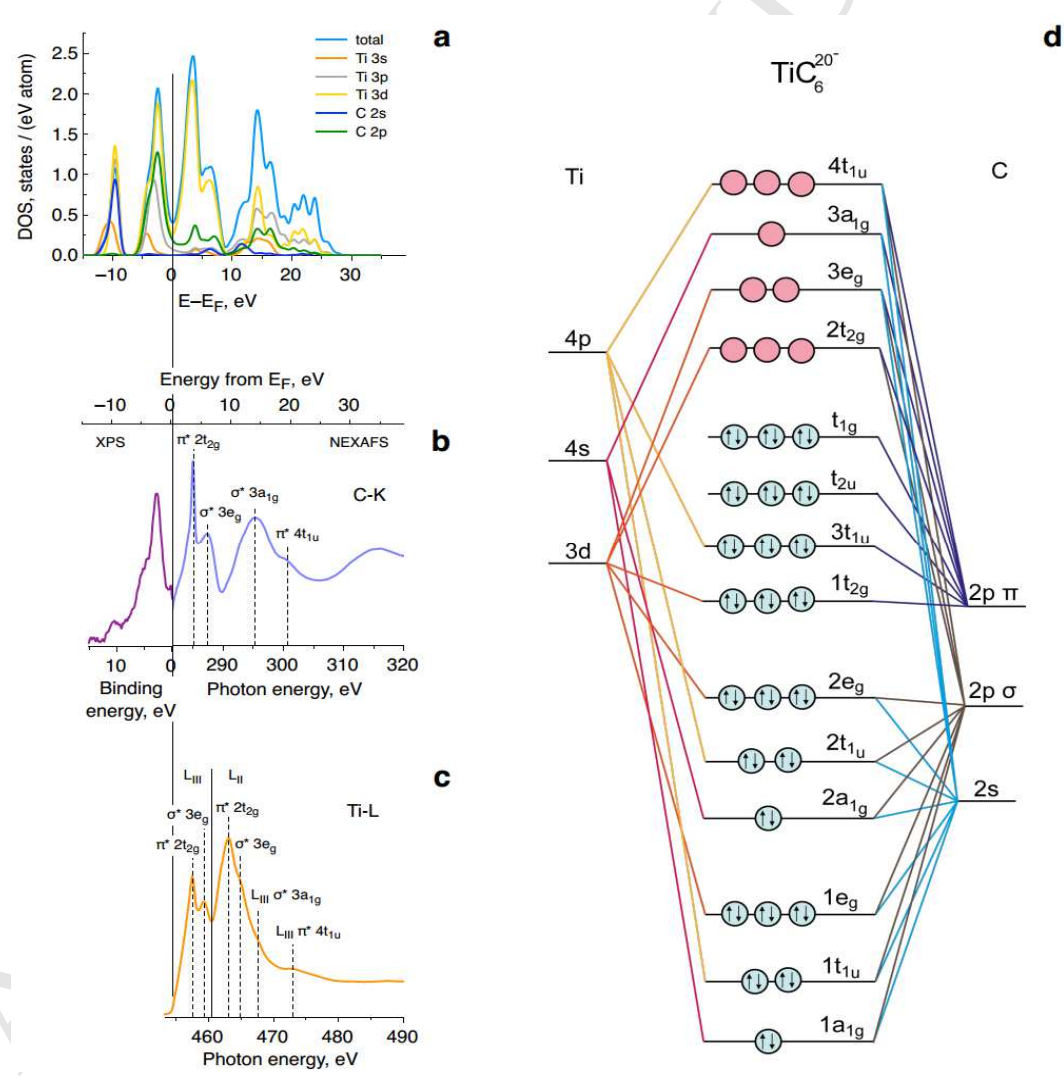

Figure 2. a) DOS for the bulk TiC as calculated by DFT, b) the C- $K$ absorption spectrum (right) and the valence band spectrum (left) for the $\mathrm{TiC}$ (111) surface, c) the $\mathrm{Ti}-L$ absorption spectrum for the $\mathrm{TiC}$ (111) surface, d) molecular orbitals for cluster $\mathrm{Ti}_{6}{ }^{20-}[21]$.

For the Ti- $L$ absorption spectrum in Fig. 2c the spin-orbit doublet structure should be taken into account, since it screens the features at energies higher than $10 \mathrm{eV}$ above the Fermi 
level. Furthermore, the NEXAFS spectroscopy will be applied as a complimentary method to photoemission spectroscopy in order to prove the graphene formation and to probe the nature of interaction between $\mathrm{TiC}$ and graphene grown on its surface.

\subsubsection{Photoemission spectra: background and plasmons}

The X-ray photoemission spectra are presented in Fig. 3 for TiC (755) and in Fig. S1 of the Supporting information for TiC (111). For the correct interpretation of the background we recorded the spectra in a wide range of binding energies. The background was described by Tougaard three-parameter universal cross section for transition metals using parameters $C=1000, D=1330$ as determined from the REELS data [22], whereas $B$ was varied since it may be influenced by photoelectron diffraction effects. This procedure, however, does not provide a proper description of plasmon losses for the metallic TiC. Plasmons are caused by interaction of the emitted electrons with free electron gas. During photoemission free electrons are excited into specific states that can be described as collective oscillations with certain characteristic frequencies, as shown previously for different metals, e.g. $\mathrm{Al}$ [23], $\mathrm{Mg}$ [24], Ag, Au [25] etc.

As clearly seen in Fig. 3, the plasmon structure is pronounced both in $\mathrm{C} 1 s$ and $\mathrm{Ti} 2 p$ spectra. In detail, for the C $1 s$ core level spectrum in Fig. 3a the plasmon peaks are wellresolved. This is clearly seen in the upper inset. To distinguish between the bulk and surface plasmon positions we used the EELS data reported earlier in Ref [16]. Plasmon losses are marked in Fig. 3a. The peaks corresponding to the surface plasmons are at binding energies lower than the corresponding bulk plasmon approximately by $\sqrt{ } 2$ times. The spectral features are summarized in Table 2. The same plasmon loss features can be found in Ti $2 s$ spectrum, however, they are much less intense (see Supporting Information, Fig. S2). For the Ti $2 p$ spectrum in Fig. $3 \mathrm{~b}$ the situation is more complicated because of the overlaying losses that correspond to the $2 p_{3 / 2}$ and $2 p_{1 / 2}$ spin orbit components. Nevertheless, the plasmon peaks are located at the same binding energies with respect to the main $2 p_{3 / 2}$ and $2 p_{1 / 2}$ peaks. One should stress that the plasmon structure for (755) is the same as that for the (111) surface, thus demonstrating negligible effect of the atomic steps. It is important to note that the intensity of the plasmon peaks is not included into quantification of the surface composition.

Table 1. A summary of the features in the NEXAFS spectra and the valence band presented in Fig. 2. 


\begin{tabular}{|c|c|c|c|c|}
\hline Feature & $\begin{array}{l}\text { Photon } \\
\text { energy/ } \\
\text { binding } \\
\text { energy, } \\
\text { eV }\end{array}$ & $\begin{array}{l}\text { Energy from } E_{F} \text {, } \\
\text { eV }\end{array}$ & $\begin{array}{l}\text { Assignment from } \\
\text { molecular orbitals } \\
\text { for the } \mathrm{TiC}_{6}{ }^{20-} \text { cluster } \\
{[21]}\end{array}$ & $\begin{array}{l}\text { The calculated DOS } \\
\text { feature and } \\
\text { assignment, eV }\end{array}$ \\
\hline \multirow{3}{*}{$\begin{array}{l}\text { Occupied } \\
\text { states } \\
\text { Valence } \\
\text { band }\end{array}$} & $9.6-12$ & $9.6-12$ & $\begin{array}{l}l e_{\mathrm{g}}=\mathrm{C} 2 s+\mathrm{Ti} 3 \mathrm{~d} \\
1 t_{1 \mathrm{u}}=\mathrm{C} 2 s+\mathrm{Ti} 4 \mathrm{p} \\
l a_{1 \mathrm{~g}}=\mathrm{C} 2 s+\mathrm{Ti} 4 \mathrm{~s}\end{array}$ & $\begin{array}{l}-10.7 \mathrm{eV}(\mathrm{Ti} 4 s) \\
-9.7(\mathrm{Ti} 4 p 3 d+\mathrm{C} \\
2 s)\end{array}$ \\
\hline & $4.5-6.4$ & $4.5-6.4$ & $\begin{array}{l}2 e_{\mathrm{g}}=\mathrm{C} 2 p \sigma+\mathrm{Ti} 3 \mathrm{~d} \\
2 t_{1 \mathrm{u}}=\mathrm{C} 2 p \sigma+\mathrm{Ti} 4 \mathrm{p} \\
2 a_{1 \mathrm{~g}}=\mathrm{C} 2 s+\mathrm{Ti} 2 \mathrm{~s}\end{array}$ & $\begin{array}{l}-3.8(\mathrm{Ti} 4 s 3 d+\mathrm{C} \\
2 p)\end{array}$ \\
\hline & 2.9 & 2.9 & $\begin{array}{l}t_{1 \mathrm{~g}}=\mathrm{C} 2 \mathrm{p} \pi ; t_{2 \mathrm{u}}=\mathrm{C} \\
2 \mathrm{p} \pi ; 3 t_{1 \mathrm{u}}=\mathrm{C} 2 \mathrm{p} \\
\pi+\mathrm{Ti} 4 \mathrm{p} ; 1 t_{2 \mathrm{~g}}=\mathrm{C} 2 \mathrm{p} \\
\pi+\mathrm{Ti} 3 \mathrm{~d} ;\end{array}$ & $\begin{array}{l}-2.4(\mathrm{Ti} 4 p 3 d+\mathrm{C} \\
2 p)\end{array}$ \\
\hline \multirow{4}{*}{$\begin{array}{l}\text { Unoccupied } \\
\text { states } \\
\text { C- } K\end{array}$} & 285.0 & 3.6 & $\pi^{*} 2 t_{2 \mathrm{~g}}=\mathrm{Ti} 3 \mathrm{~d}+\mathrm{C} 2 p$ & $\begin{array}{l}3.1,3.7(\mathrm{Ti} 3 d+ \\
\text { C } 2 p)\end{array}$ \\
\hline & 287.3 & 5.9 & $\begin{array}{l}\sigma^{*} 3 e_{g}=\mathrm{Ti} 3 d+\pi \mathrm{C} \\
2 p\end{array}$ & $\begin{array}{l}5.6,6.6(\mathrm{Ti} 3 d+\mathrm{C} \\
2 p)\end{array}$ \\
\hline & 295.3 & 13.9 & $\begin{array}{l}\sigma^{*} 3 a_{1 g}=\mathrm{Ti} 4 s+\pi \mathrm{C} \\
2 p\end{array}$ & $\begin{array}{l}14.2(\mathrm{Ti} 4 p 3 d+\mathrm{C} \\
2 p)\end{array}$ \\
\hline & 300.5 & 19.1 & $\begin{array}{l}\pi^{*} 4 t_{1 u}=\mathrm{Ti} 4 s+\pi \mathrm{C} \\
2 p\end{array}$ & $16.5(\mathrm{Ti} 4 p+\mathrm{C} 2 p)$ \\
\hline \multirow{4}{*}{$\begin{array}{l}\text { Unocuppied } \\
\text { states } \\
\text { Ti-L }\end{array}$} & 457.5 & 3.3 & $L_{\mathrm{III}} \pi * 2 t_{2 g}$ & $\begin{array}{l}3.1,3.7(\mathrm{Ti} 3 d+\mathrm{C} \\
2 p)\end{array}$ \\
\hline & 459.5 & 5.3 & $\mathrm{~L}_{\mathrm{III}} \sigma^{*} 3 e_{g}$ & $\begin{array}{l}5.6,6.6(\mathrm{Ti} 3 d+\mathrm{C} \\
2 p)\end{array}$ \\
\hline & 463.2 & 9.0 & $\mathrm{~L}_{\mathrm{II}} \pi^{*} 2 t_{2 g}$ & - \\
\hline & 465.2 & 11.0 & $\mathrm{~L}_{\mathrm{II}} \sigma^{*} 3 e_{g}$ & \\
\hline
\end{tabular}

\subsubsection{Photoemission spectra: Shake-up satellite}

The main Ti $2 p$ doublet has a complex shape. First, the $2 p_{1 / 2}$ to $2 p_{3 / 2}$ intensity ratio is not $1: 2$; it depends on electron kinetic energies. In detail, the kinetic energies of $2 p_{3 / 2}$ electrons of $200,150,100,56 \mathrm{eV}$ show the ratios of $0.66,0.65,0.48$ and 0.87 , correspondingly. We suppose that this ratio is strongly influenced by photoelectron diffraction. Second, detailed inspection reveals that this doublet cannot be fitted satisfactory using a single doublet of Gaussian-Lorenzian convolution peak shape even after introducing the Doniah-Sunjic peak asymmetry and treating the contamination-related features accurately. The peak asymmetry seems to be reasonably low $(\alpha=0.08)$ taking into account the low electron density of states at the Fermi level. 
a

C 1s

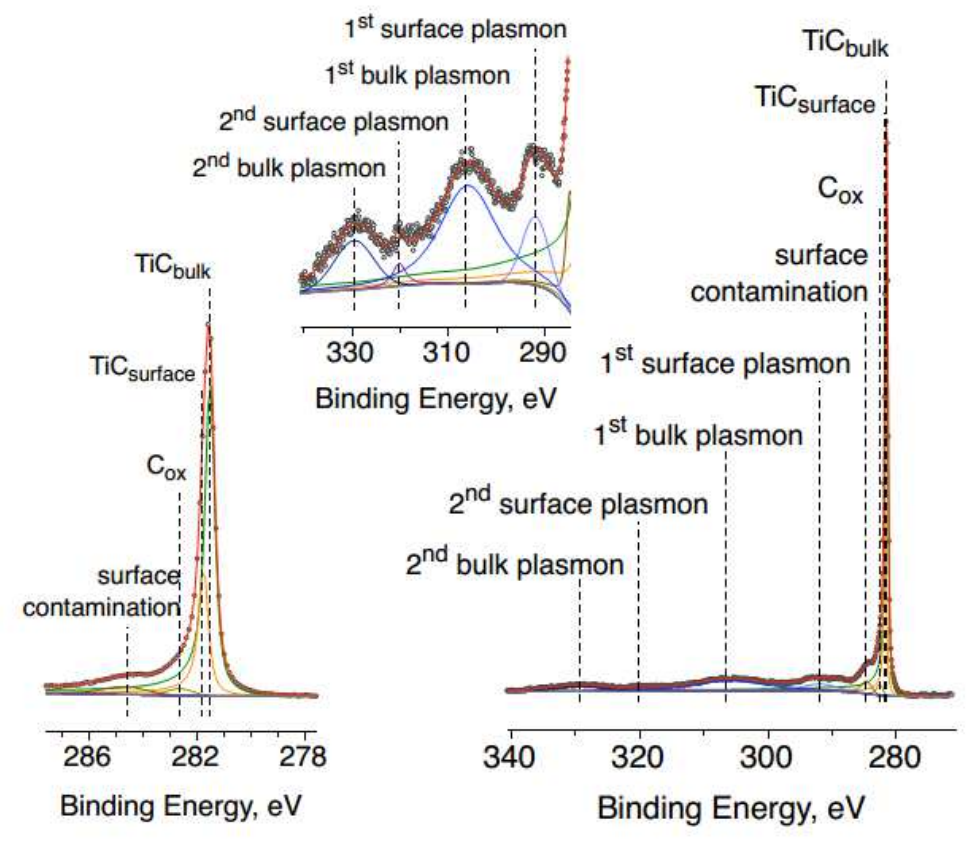

b

Ti $2 p$

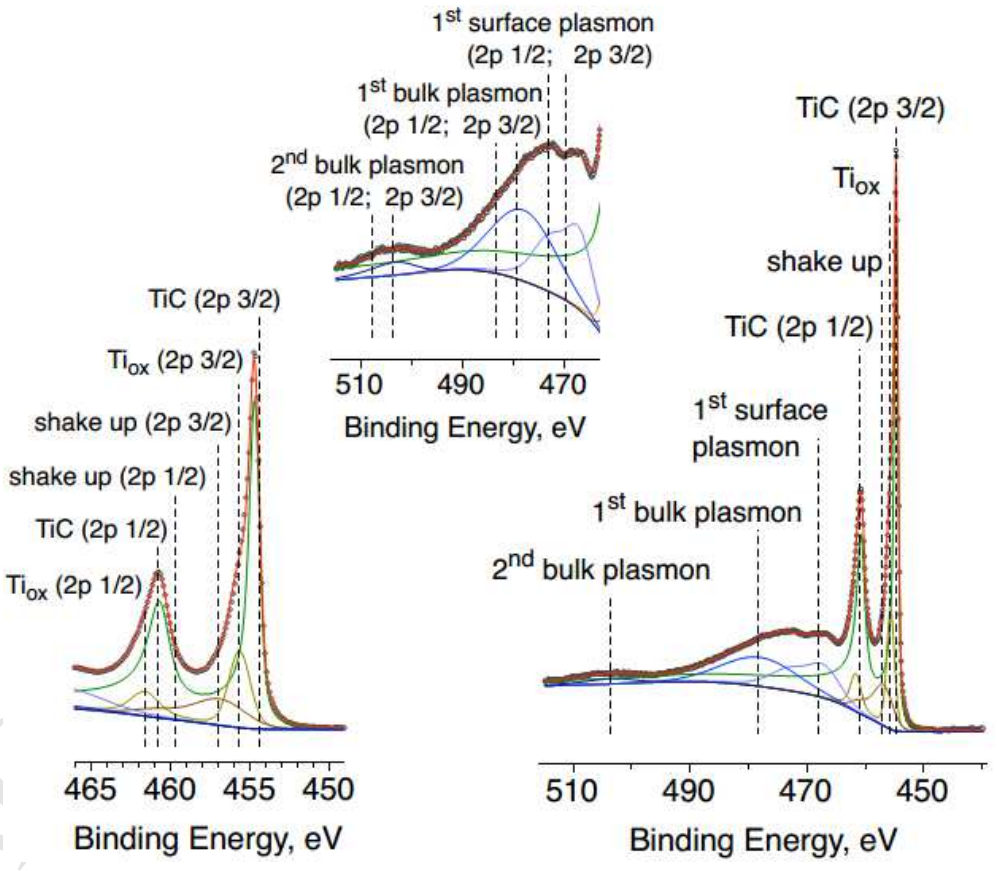

Figure 3. C $1 s$ (a), and Ti $2 p$ (b) X-ray photoemission spectra for the clean TiC (755) surface obtained at an electron kinetic energy of $200 \mathrm{eV}$ in a wide range of binding energies. The inserts show the plasmon structure and the main peak shape.

One of the possible contributions that have not yet been discussed above is a shake-up satellite. Strong satellites have been observed earlier in photoemission spectra of $3 d$ metals 
and some of their compounds like TiN [13, 14]. According to Hüfner [26], a shake-up is a peak with lower kinetic energy than that of the main line, which corresponds to a discrete intrinsic electron-electron interaction. It is described by the Kotani-Toyozawa model [27]. According to this model, for $3 d$ metals the coulomb interaction of the core photohole with the valence $d$ band leads to a situation when an empty $d$ level is pulled below the valence band maximum, thus giving formally an additional positive charge. This state is called a two-hole state (a core photohole plus a hole due to the empty $d$ level). It can be screened in two different ways: (i) by a charge moving rapidly from the broad $s p$ conduction band or (ii) by a charge transferring from the $s p$ band to the lowered $d$ level. The system response to the core hole is instantaneous and will result in two final states of the emitted photoelectron: a ground state (the main peak) and an excited state (the shake-up). Since the localized $d$ level is more efficient in screening than the extended $s p$ band, this second final state has a lower binding energy than the first one. The second scenario results in the main photoemission peak, whereas the first mechanism gives rise to a shake-up satellite. In our case these two final states correspond to (i) the screening of the $3 d^{2}$ hole by Ti $4 s^{2}$ and $\mathrm{C} 2 s^{2} 2 p^{2}$ electrons or (ii) the filling of the $3 d^{2}$ hole with $\mathrm{Ti} 4 s^{2}$ or $\mathrm{C} 2 s^{2} 2 p^{2}$ electrons.

There are different ways to figure out whether the shake-up satellite contributes to the spectrum [28]. One of them is resonant photoemission which is excited by a photon with an energy corresponding to a certain electron transition [29]. In our case one can use the Fano resonance conditions, when an electron emitted from the valence band interferes with the Auger $C V V$ electrons at a photon energy corresponding to an electron transition from $3 p$ to $3 d$ orbital. In this case after the relaxation the final state is the same as for the shake up satellite. It can be described by the following equations:

$$
\begin{gathered}
h v+3 p^{6} 3 d^{2} \rightarrow 3 p^{5} 3 d^{3} \rightarrow 3 p^{6} 3 d^{1}[4 s]+e^{-}\left(E_{k i n}=E_{3 p}-E_{\text {sat }}\right), \\
h v+3 d^{2} \rightarrow 3 d^{1}[4 s]+e^{-}\left(E_{k i n}=h v-E_{s a t}\right),
\end{gathered}
$$

where $h v$ is the photon energy, $E_{k i n}$ is the kinetic energy of the photoelectron, $E_{s a t}$ is the satellite energy.

According to the resonant photoemission data obtained for TiC in Ref. [15], the Fano resonance is indeed revealed at energy loss of $2-3 \mathrm{eV}$ relative to the $3 d$ peak; however, it is difficult to determine its exact energy because the resonance is much weaker than that for pure titanium.

To sum up, if an additional feature is introduced into the Ti $2 p$ spectra, the resulting peak has a relative intensity of $7 \%$ and is split by $2.2 \mathrm{eV}$ from the main line. We assign it to 
the shake-up satellite described above. Compared to the spectrum for TiN, the shake-up peak intensity is essentially lower in our case due to the difference in the $3 d$ band population (formal $\mathrm{Ti}^{4+}$ against formal $\left.\mathrm{Ti}^{3+}\right)[1]$ and lower electron density of states at the Fermi level.

One should stress that all spectral features for the (755) and (111) surfaces a fully identical. Their comparison is given in Table 2.

\subsubsection{Photoemission spectra: surface core level shift (SCLS)}

One of the typical features for clean surfaces of many metals and ionic crystals is the so-called surface core-level shift (SCLS), which is revealed due to the fact that the electron density of the undercoordinated atoms at the surface differs from that of the bulk atoms even in the absence of any surface reconstruction. Detailed analysis of the Ti $2 p$ and $\mathrm{C} 1 s$ spectra obtained at the ultimate resolution demonstrates that the SCLS of $+0.25 \mathrm{eV}$ does occur for carbon atoms. The spectra obtained for the (755) and (111) surfaces are exhibited in Fig. 4a,b. In detail, both $\mathrm{C} 1 s$ spectra contain two components split by $0.25 \mathrm{eV}$ and their ratio depends on the emission angle (Fig. 4c) and the photon energy (Fig. 4d). This data supports the surface nature of the component at higher binding energy, which can be proven by comparing spectra obtained at different electron kinetic energies and electron emission angles. One should stress that there is no notable difference between the spectra obtained for the (111) and (755) surfaces, which evidences minor contribution of the step edge atoms. Precise quantitative analysis is impossible since the fast surface contamination influences the surface-to-bulk intensity ratio in the UHV at the base pressure of $410^{-10} \mathrm{mBar}$. In the Ti $2 p$ spectra the SCLS, if it exists, cannot be resolved.

As mentioned above, both $\mathrm{TiC}(111)$ and $\mathrm{TiC}$ (755) surfaces are polar and in our case a Ti-termination is typical for our surface preparation procedure [19]. According to the calculations reported in Ref.[11, 20], the Ti terminated surface attains a relatively large positive charge of 0.8 electron per atom. Surface formation is accompanied by strong contraction of the first interatomic distance, by $17 \%$ in our DFT calculations (see the inset in Fig.4b and Fig.S3 of Supporting information), which, however, does not provide complete charge compensation at the surface. Higher binding energy for the atoms in the upper carbon layer, therefore, is due to the weaker charge transfer from the surface $\mathrm{Ti}$ atoms to the subsurface $\mathrm{C}$ atoms as compared to the charge transfer in the bulk TiC. The calculated SCLS for $\mathrm{C} 1 s$ is $+0.33 \mathrm{eV}$ and for Ti $2 p$ is $+0.43 \mathrm{eV}$ for 12 layer slab modelling TiC (111) surface (SI, Fig. S3). This is in reasonable agreement with the experimental observations. 
Table 2. A summary of XP-spectral features for clean TiC (755) and TiC (111) surfaces

\begin{tabular}{|c|c|c|c|c|}
\hline \multirow[t]{2}{*}{ Peak and assignment } & \multicolumn{2}{|c|}{ Binding energy, eV } & \multicolumn{2}{|c|}{ Relative intensity, \% } \\
\hline & TiC (755) & TiC (111) & TiC (755) & $\mathrm{TiC}(111)$ \\
\hline \multicolumn{5}{|c|}{ C 1s } \\
\hline $\mathrm{TiC}_{\text {bulk }}$ & 281.5 & 281.8 & 46.3 & 41.4 \\
\hline $\mathrm{TiC}_{\text {surface }}(\mathrm{SCLS})$ & $281.75(+0.25)$ & $\begin{array}{l}282.11 \\
(+0.31)\end{array}$ & 18.7 & 26.5 \\
\hline Oxidized species & $282.6(+1.1)$ & $282.8(+1.0)$ & 2.2 & 1.3 \\
\hline $\begin{array}{l}\text { Adventitious } \\
\text { contamination }\end{array}$ & $284.6(+3.1)$ & $284.4(+2.6)$ & 3.8 & 0.6 \\
\hline $1^{\text {st }}$ surface plasmon & $291.9(+10.4)$ & $292.4(+10.6)$ & 4.9 & 4.6 \\
\hline $1^{\text {st }}$ bulk plasmon & $305.9(+24.4)$ & $305.2(23.4)$ & 17.8 & 14.7 \\
\hline $2^{\text {nd }}$ surface plasmon & $320.2(+38.7)$ & $319.1(+37.3)$ & 1.2 & 3.3 \\
\hline $2^{\text {nd }}$ bulk plasmon & $329.5(+48.0)$ & $331.5(+49.7)$ & 5.3 & 7.7 \\
\hline \multicolumn{5}{|c|}{ Ti $2 s$} \\
\hline $\mathrm{TiC}$ & 560.2 & 562.2 & 60.6 & 54.1 \\
\hline Oxidized species & $561.1(+1.0)$ & $\sqrt{2+1}$ & 8.7 & \\
\hline shake up & $562.4(+2.2)$ & $564.4(+2.2)$ & 2.9 & 4.1 \\
\hline surface plasmon & $582.5(+12.3)$ & $574.2(+12.0)$ & 15.1 & 14.6 \\
\hline $1^{\text {st }}$ bulk plasmon & $582.5(+22.3)$ & $586.6(+24.4)$ & 3.7 & 15.1 \\
\hline $2^{\text {nd }}$ bulk plasmon & $608.3(+48.1)$ & $611.0(+48.8)$ & 9.1 & 11.32 \\
\hline \multicolumn{5}{|c|}{ Ti $2 p$} \\
\hline $\mathrm{TiC}$ & 454.8 & 455.0 & 53.7 & 49.7 \\
\hline Oxidized species & $455.8(+1.0)$ & $455.9(+0.9)$ & 10.5 & 15.7 \\
\hline shake up & $457.0(+2.2)$ & $457.2(+2.2)$ & 6.7 & 7.0 \\
\hline surface plasmon & $467.1(+12.3)$ & $467.0(+12.0)$ & 12.7 & 19.1 \\
\hline $1^{\text {st }}$ bulk plasmon & $477.1(+22.3)$ & $479.4(+24.4)$ & 14.6 & 8.2 \\
\hline $2^{\text {nd }}$ bulk plasmon & $502.9(+48.1)$ & $503.9(+48.9)$ & 1.9 & 0.3 \\
\hline
\end{tabular}


a
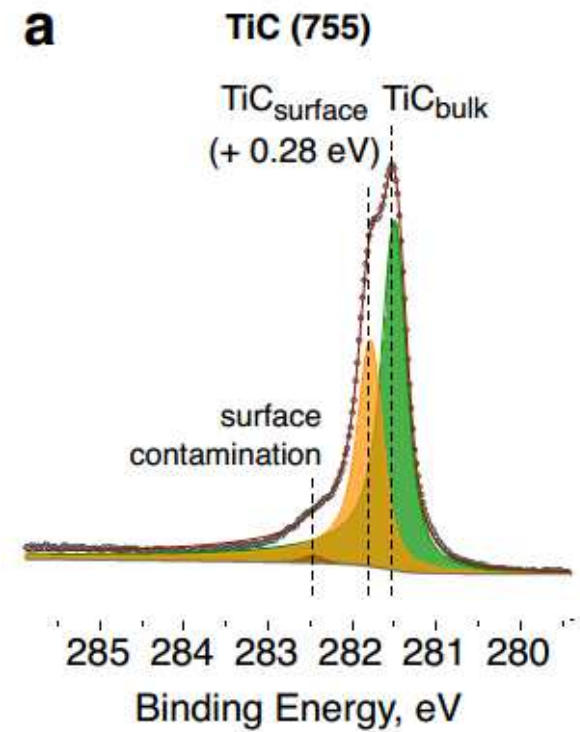

C

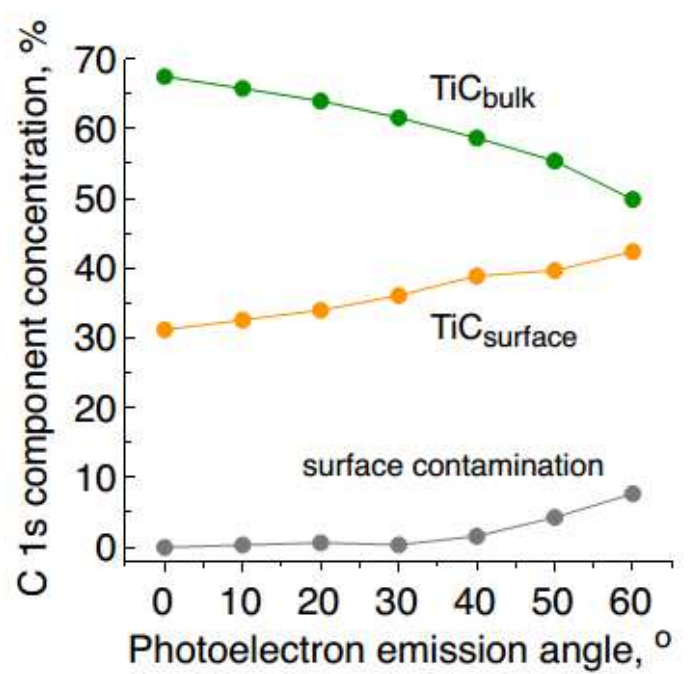

b $\quad$ TiC (111)

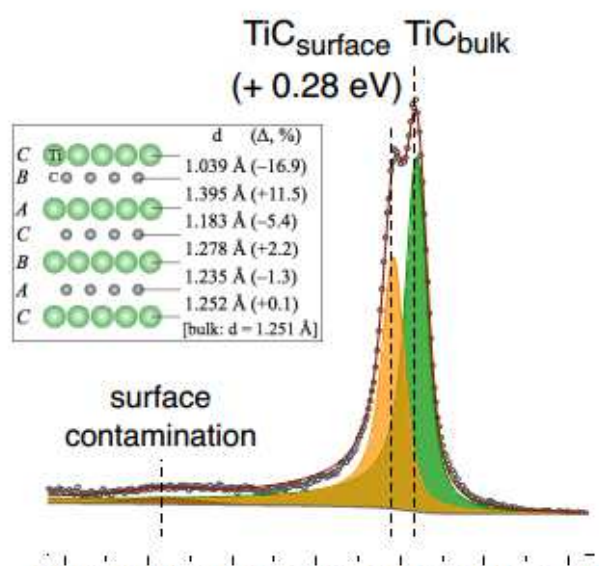

$286286^{2} 284283282281280$ Binding Energy, eV

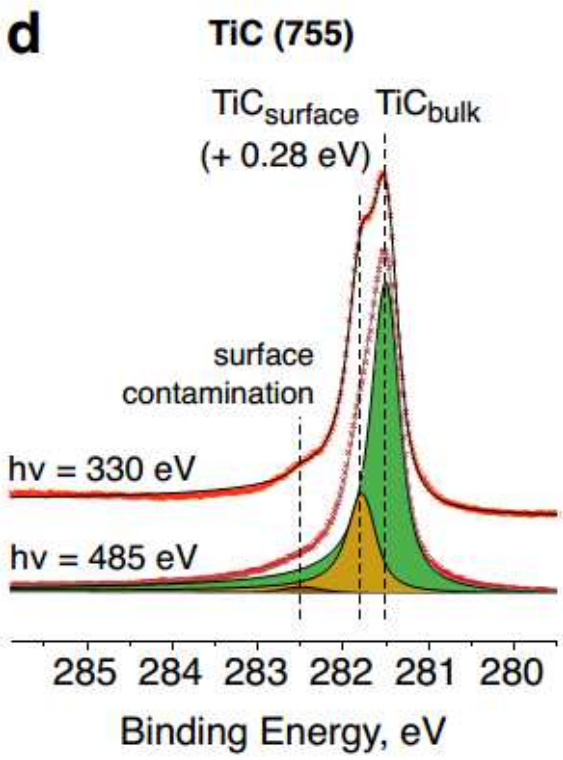

Figure 4. High resolution C $1 s$ photoemission spectra for the $\mathrm{TiC}$ (755) (a) and (111) (b) surfaces at a photon energy of $330 \mathrm{eV}, \mathrm{c}$ ) angular dependence of the component ratio for a photon energy of $485 \mathrm{eV}$ in $\mathrm{C} 1 \mathrm{~s}$ spectra for $\mathrm{TiC}(755)$, d) C 1s spectra obtained for TiC (755) at two different kinetic energies, 45 and $200 \mathrm{eV}$. Surface contamination peak, not avoidable at 4.10-10 mbar base pressure, is treated using parameters determined from oxidation behavior. The insert in (b) shows the calculated atomic geometry for the TiC (111) surface (see SI for details).

To sum up the XP-spectral features, we can conclude that for the clean TiC (111) and (755) surfaces the photoemission spectra demonstrate bulk and surface plasmons, shake-up satellites revealed in the Ti $2 p$ and Ti $2 s$ spectra, and the surface core-level shift of $0.25 \mathrm{eV}$ in the C $1 s$ spectra. 


\subsection{Graphene on $\mathrm{TiC}(111)$ and (755)}

\subsubsection{Structure}

As a result of the CVD growth, graphene layers were obtained both on the (111) and (755) surfaces. The angular dispersion of the C- $K$ absorption spectra in Fig. 5 - as received (a) and after subtraction of the substrate signal (b) - evidences the formation of a flat layer of $s p^{2}$-carbon. For the latter the peak intensity related to the $\pi^{*}$-state transition in graphene decreases when the angle between the polarization vector of the synchrotron light and the surface plane increases; it vanishes at $90^{\circ}$. In addition, we found no dispersion in the Ti- $L$ spectra (as shown in Fig.S4 of Supporting Information).
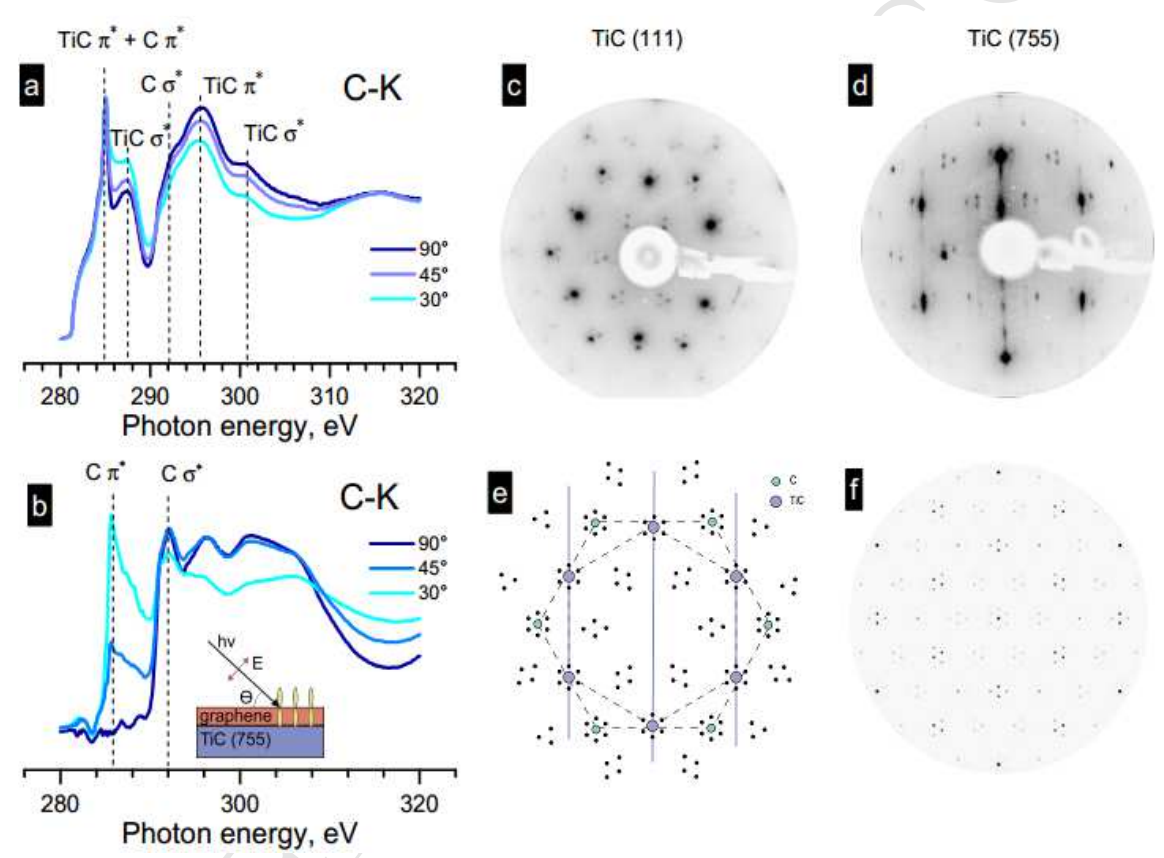

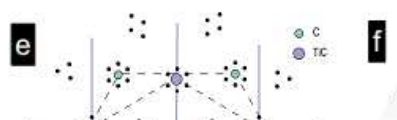

$\therefore \quad: \quad \therefore \quad \therefore$

$\therefore \quad \therefore \quad \therefore \quad \therefore$

$\because \therefore: \therefore \quad \therefore \quad \because$

$\therefore \quad: \quad:-a$

$: \quad: \quad$

Figure 5. Graphene on the $\mathrm{TiC}$ (755) surface: the $\mathrm{C} K$ absorption spectra obtained at different incidence angles (a) and after the substrate spectrum subtraction (b). LEED patters obtained for graphene on the (111) (c) and (755) (d) surfaces at an electron energy of $125 \mathrm{eV}$; schematic representation of the experimental LEED pattern (e) and modeling results for the (111) surface (f).

Compared to the clean surfaces, the LEED patterns for graphene grown on (111) and (755) surfaces presented in Fig. 5c,d reveal additional reflexes, i.e. superstructure spots. Fig. 5e summarizes all features observed in the LEED patterns obtained at different energies. 
To determine the surface structure, we simulated the LEED pattern for the (111) surface as shown in Fig. 5f. In our model we assumed that graphene is corrugated according to the previous STM observations [3] with a peak-to-peak amplitude of $0.5 \AA$ and a period of TiC (111) (3.06 ̊). The closest commensurate approximation to the graphene/TiC (111) structure was found to be $(7 \sqrt{ } 3 \times 7 \sqrt{ } 3) \mathrm{R} 30^{\circ}$.

For the (755) surface the LEED pattern in Fig. $5 \mathrm{~d}$ can be easily presented as a superposition of graphene and the $\mathrm{TiC}$ (755) $1 \times 1$ patterns rotated by $30^{\circ}$ with respect to each other and a number of superstructure spots [3]. The absence of any streaks near the spots from the layer indicates that graphene forms a continuous layer rather than ribbons.

\subsubsection{Graphene-substrate interaction and band structure}

The photoemission spectra for graphene covered (755) and (111) surfaces are presented in Fig. 6a and 6b correspondingly. In the Ti $2 p$ spectra there are no remarkable changes upon deposition (for details refer to Fig. S5 of the Supporting information file). In the $\mathrm{C} 1 s$ spectra we observed an additional pronounced feature related to graphene. It is rather broad (FWHM is $0.6 \mathrm{eV}$ ), i.e. much broader than that for graphene on $\operatorname{Ir}(111)$ (FWHM is $0.3 \mathrm{eV}$ ) [30], for which the distance between carbon and $\mathrm{Ir}$ atoms is different due to corrugation. The peak maximum is positioned at a binding energy of $285 \mathrm{eV}$, which is $0.5 \mathrm{eV}$ higher than that of free-standing graphene. This fact already pinpoints some kind of interaction between graphene and the substrate, which seems to be varying for different graphene atoms taking into account the large peak width. It should be noted that the work function of $\mathrm{TiC}$ is very close to that typical for undoped graphene [31, 32], therefore, we do not anticipate any charge redistribution between graphene and the substrate within the rigid band model. As discussed above, the Ti-terminated TiC (111) surface is positively charged with the corresponding net charge of +0.8 electron per atom according to the calculations [11]. The graphene layer on such a surface can also acquire a positive charge. This, at first sight, fits the experimental observation of the positive binding energy shift. However, in the case of pure electrostatic interaction the subsurface carbon layer should acquire a more negative charge compared to the clean surface, since the electron density is partially shifted from graphene to the surface $\mathrm{Ti}$ atoms. This is definitely not the case as one can clearly see that the SCLS in the C $1 s$ spectra becomes notably larger, by $0.1 \mathrm{eV}$, for the graphenecovered surface. The reason for such increase of the SCLS value upon graphene growth remains unclear. If it is not due to electrostatic interaction, then it can be explained solely by hybridization between graphene and the substrate. This is in line with the larger peak width 
of the C $1 s$ spectra for our system than that for the $\operatorname{Ir}(111)$ substrate where the coupling is negligible. The hybridization concept is also supported by the C- $K$ absorption spectra shown in Fig.5b, which differ from those for the free-standing graphene. In detail, additional spectral features are observed between the $\pi$ and $\sigma$ resonances. As already investigated experimentally [33] and theoretically [34] for graphene grown on $\mathrm{Ni}$, these features indicate hybridization between graphene and substrate. Here, again we found no notable difference between the spectra obtained for the (111) and (755) surfaces, which evidences the negligible effect of the atomic steps.

In order to learn more about chemical peculiarities of interaction between graphene and the TiC surface, we studied the band structure of the graphene/TiC (755) by means of angle-resolved photoelectron spectroscopy (ARPES). Fig. 7a shows the resulting band structure of the graphene-covered TiC (755) measured along high symmetry directions $\overline{\Gamma \mathrm{M}}$ and $\overline{\Gamma K}$ of the graphene surface Brillouin zone (SBZ). Traces that indicate the direction for the dispersion measurement are sketched in the inset depicting SBZ by red lines.

The overall band structure in Fig. 7a shows that the $\pi$-band of graphene (labelled in the figure as $\mathrm{gr}-\pi$ ) is strongly shifted toward higher binding energies as compared to the electronic structure of freestanding graphene $[35,36]$. Furthermore, this shift is non-rigid and is different in various high-symmetry points of the SBZ. Extrema of the graphene band dispersions are denoted in Fig. 7a with blue horizontal ticks and labelled with exact values of their binding energy. One can see that the deviation from the ideal freestanding graphene at $\bar{\Gamma}$ point is about $1.5 \mathrm{eV}$. At the borders of the SBZ the energy shifts toward higher binding energy are much larger. At the $\bar{M}$ point [left part of Fig. 7(a)] the measured shift of graphene band, determined as a medium energy between bonding and antibonding states $\pi$ and $\pi^{*}$, is about $3 \mathrm{eV}$. This value can be determined precisely since the shift is so large that $\pi^{*}$ (labelled in Fig. 7 as gr- $\pi^{*}$ ) moves below the Fermi level and can be accessed by ARPES. 

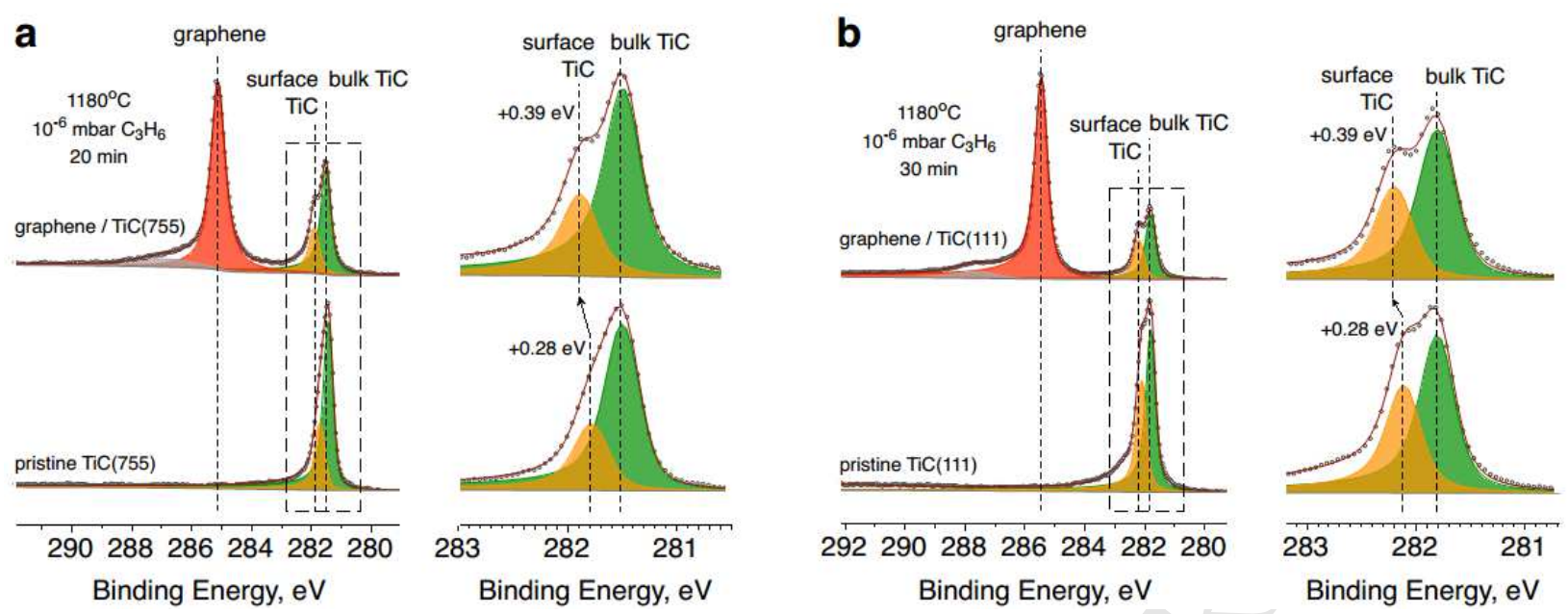

Figure 6. C 1s photoemission spectra for the pristine and graphene covered $\mathrm{TiC}$ (755) (a) and (111) (b) surfaces recorded at an electron kinetic energy of $200 \mathrm{eV}$.

The energy shift at the $\overline{\mathrm{K}}$ point can be determined from the measurements of the band structure performed along the direction perpendicular to $\overline{\Gamma K}$ of the graphene SBZ. Such geometry allows one to observe both sides of the $\pi$ band due to the Brillouin zone effects that affect the photoelectron interference [37]. Dispersion of the Dirac bands within the energymomentum space outlined with a red dashed rectangle in Fig. 7a, but measured perpendicular to $\overline{\Gamma K}$ and precisely through the $\overline{\mathrm{K}}$ point, is shown in Fig. 7b. Surprisingly, in spite of the strong interaction between graphene and $\mathrm{TiC}$, one sees a perfectly intact and gapless Dirac cone with the Dirac point located at the binding energy of $2.35 \mathrm{eV}$. Considering that in the ideal graphene Dirac and Fermi energies coincide, we define this value as a magnitude of the energy shift at $\overline{\mathrm{K}}$.

Such giant and non-rigid energy shift of the graphene band structure is an indication of strong interaction between graphene and TiC. This interaction is largely driven by covalent bonding that depends on availability and energy position of certain states in the valence band of $\mathrm{TiC}$ and to a lesser extent by the charge transfer between graphene and its substrate [38]. The impact of orbital hybridization between graphene and $\mathrm{TiC}$ on non-rigid energy shift and the overall width of the $\pi$ band has been suggested earlier [14, 39]. In our present findings, this covalent bonding is manifested through rich hybridization effects denoted by black arrows in Fig. 7. The formation of hybridization gaps and kinks in the dispersion of $\pi$ band near the $\overline{\mathrm{K}}$ point is observed for the binding energies of $0.5-1.0 \mathrm{eV}$ (Figs. $7 \mathrm{a}$ and $7 \mathrm{~b}$ ) and in the dispersion of the $\pi^{*}$ band near the $\overline{\mathrm{M}}$ point - at the binding energy of $0.3-0.4 \mathrm{eV}$ 
(Fig. 7a). In view of strong electronic hybridization between graphene and $\mathrm{TiC}$ the intact Dirac cone observed in Fig. $7 \mathrm{~b}$ should indeed be considered as a hybrid cone with significant contributions from the TiC states - similarly to the case of intact Dirac cones observed in graphene on $\mathrm{Ni}(111)$ and $\mathrm{Co}(0001)$, which are substantially intermixed with the $3 d$ states of $\mathrm{Ni}$ and $\mathrm{Co}[40,41]$.

Another important fact which we learned from the ARPES data is lateral integrity of the graphene overlayer. Indeed, the dispersion of the $\pi$ band measured along $\overline{\Gamma K}$, which is perpendicular to the terraces of $\mathrm{TiC}$ (755), is continuous (right panel in Fig. 7b). In the case of individual graphene nanoribbons it should have fallen apart into a spectrum of discrete energy levels due to lateral quantization in nanoribbon potential wells [42, 43]. This proves that graphene covers the terraces of $\mathrm{TiC}$ (755) as a continuous carpet and not as an array of nanoribbons decorating individual terraces. On the other hand, even in the case of continuous but 1D-modulated (by the steps of $\mathrm{TiC}$ ) carpet one may expect the appearance of the renowned umklapp replica of the $\pi$ band, similar to that observed in moiré-modulated graphene on $\operatorname{Ir}(111)$ [44] and $\mathrm{Au} / \mathrm{Fe}(110)$ [45]. The absence of such replica in our ARPES data can be explained by poor lateral coherency of the graphitized TiC steps, which is also seen in the LEED pattern (Figs. 5c and d).
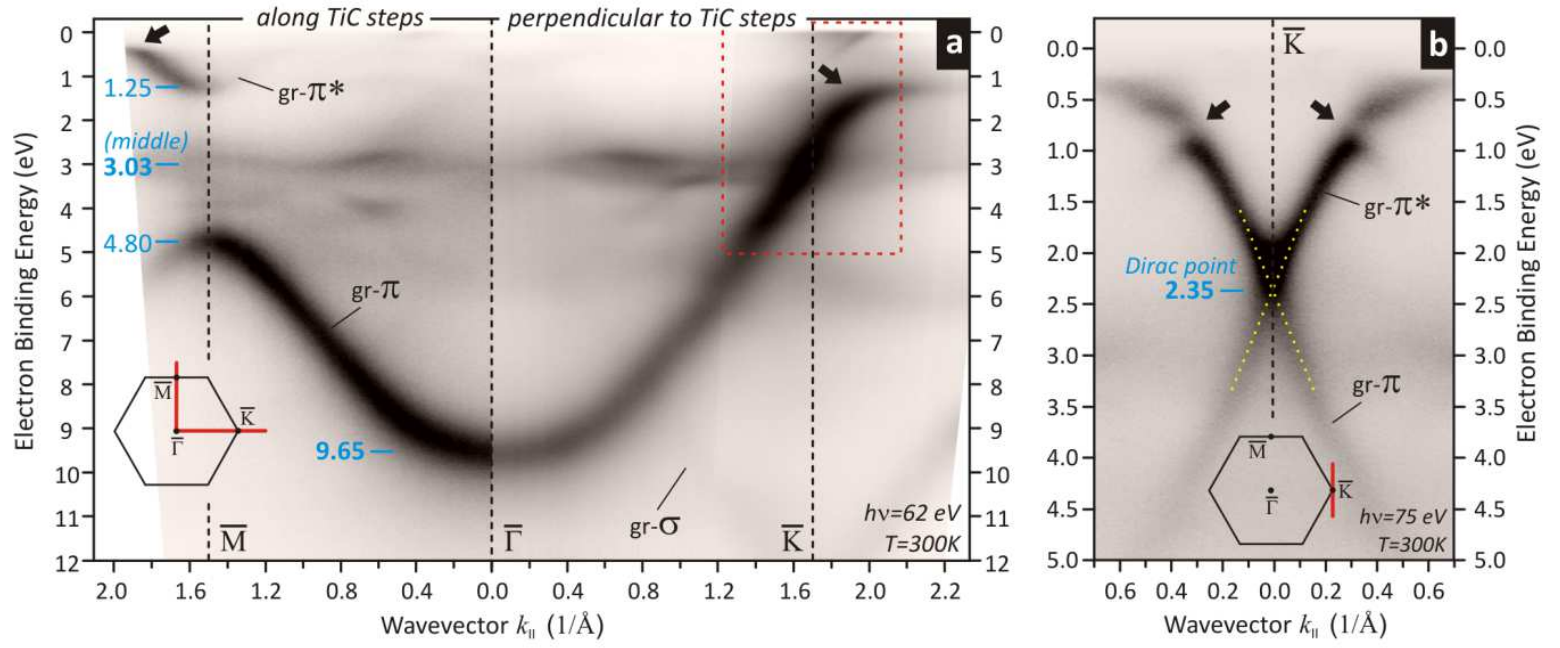

Figure 7. The electronic structure of graphene-covered TiC(755) studied by angle-resolved photoemission (ARPES): (a) the overall band structure measured along the $\overline{\Gamma M}$ and $\overline{\Gamma K}$ directions of the surface Brillouin zone (SBZ) of graphene. These directions are marked by red lines in the sketch of the SBZ in the inset. The $\overline{\Gamma K}$ direction is perpendicular to the orientation of the TiC terraces. The antibonding graphene band $\pi^{*}$ is seen at the $\overline{\mathrm{M}}$ point right below the Fermi level; (b) zoom of the graphene $\pi$ band dispersion near the $\overline{\mathrm{K}}$ point 
within the frame denoted by a red dashed rectangle in (a) but measured in the direction perpendicular to $\overline{\Gamma K}$ (the red line in the inset depicting the SBZ). At this experimental geometry both sides of the Dirac cone are visible. A perfectly intact Dirac cone is observed. Hybridization of graphene $\pi$ band with TiC (black arrows) is seen in the vicinity of the Fermi level far from the Dirac point.

\subsubsection{Surface reactivity}

Another way to check the graphene-substrate hybridization is to weaken it by intercalation of foreign atoms between graphene and the substrate. Here, we used molecular oxygen for this purpose. It is known that oxygen intercalation results in graphene and $\mathrm{Ni}$ substrate decoupling. To probe this, we exposed graphene on $\mathrm{TiC}$ (755) to oxygen at different pressures for $30 \mathrm{~min}$. We found no spectral changes up to $\sim 10^{-4}$ mbar. At higher pressures, oxygen is intercalated under graphene causing its partial decoupling and appearance of a component at $284.5 \mathrm{eV}$ corresponding to areas of free-standing graphene, which grows upon further heating. This behavior is illustrated in Fig. 8.

In addition, we revealed that a continuous graphene layer makes the $\mathrm{TiC}$ surfaces much more robust towards oxygen (at least up to $10^{-4} \mathrm{mBar}$ ), whereas a clean TiC surface is known to be rather unstable even in the UHV at a base pressure of $10^{-10} \mathrm{mBar}$, when some oxidation is already detected in the spectra acquired at a kinetic energy of $50 \mathrm{eV}$ even after a few minutes of measurements. It is commonly known that graphene forms reliable surface protection in many cases [46, 47], however, for TiC this works only at moderate vacuum conditions typical for processing. 

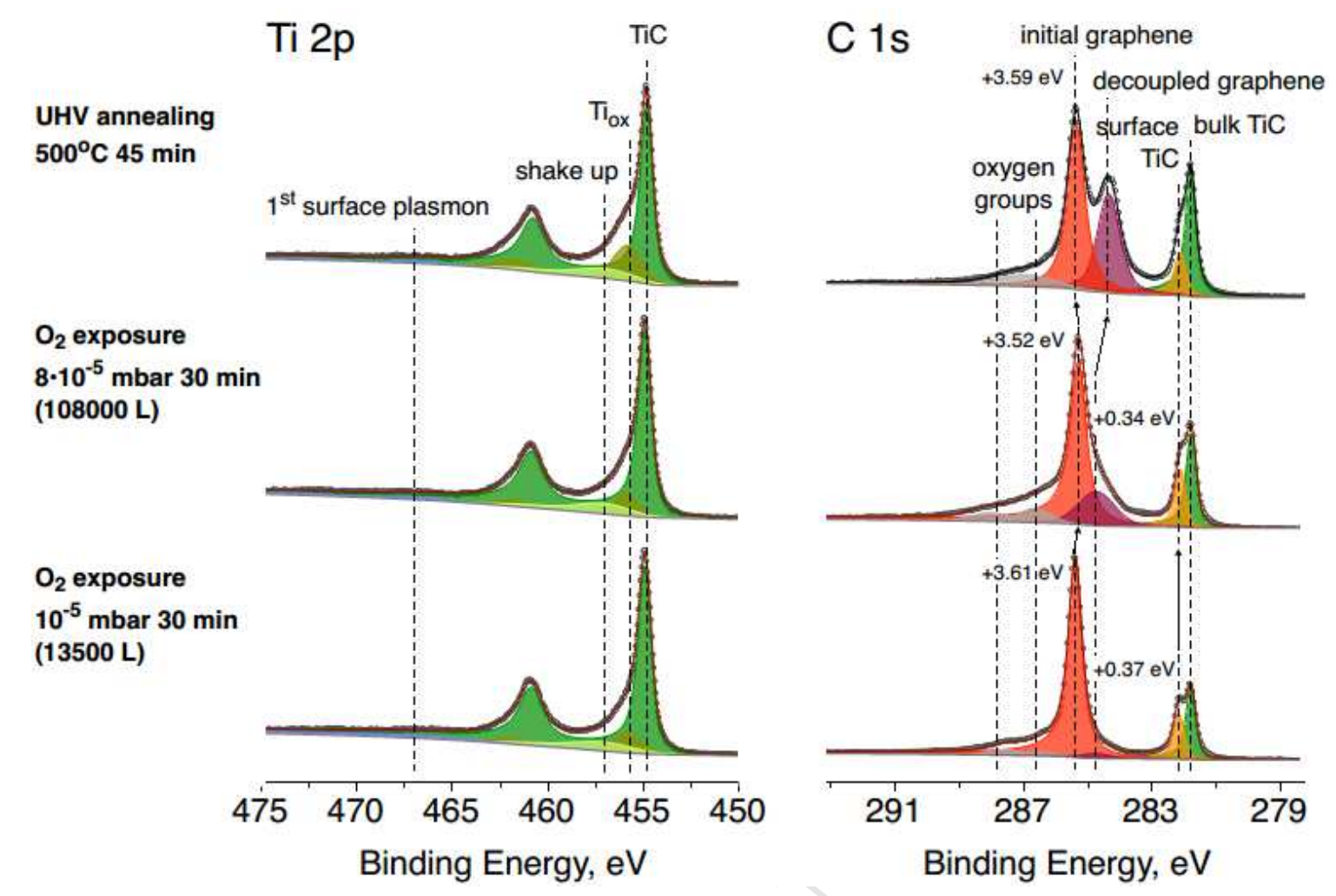

Figure 8. Photoemission spectra of the TiC (111) surface exposed to oxygen.

\section{Conclusions}

We describe comprehensively the spectral features in the XP-spectra of the clean TiC (111) and (755) surfaces including the plasmon structure, the peak asymmetry, the shake up satellite and, finally, the surface core level shift (SCLS) in C 1s spectra, which are important for further detailed studies of surface reactions involving TiC.

We synthesized graphene on both (111) and (755) surfaces and we found the SCLS variation upon graphene growth. Based on LEED data we found that the structure is close to $(7 \sqrt{ } 3 \times 7 \sqrt{ } 3) \mathrm{R} 30^{\circ}$ and graphene is slightly corrugated. In both cases graphene forms continuous layers rather than nanoribbon expected for (755) surface. Surprisingly, graphene on TiC has perfectly intact and gapless Dirac cone with Dirac point located at the binding energy of 2.35 $\mathrm{eV}$. Giant and non-rigid energy shift of graphene band structure is an indication of strong interaction between graphene and TiC. This interaction is largely driven by covalent bonding which depends on availability and energy position of peculiar states in the valence band of $\mathrm{TiC}$ and to less extent by the charge transfer between graphene and its substrate. Therefore, the SCLS variation in C 1s spectra, new features appearing in NEXAFS spectra, ARPES data and oxygen intercalation experiments, which resulted in decoupling, evidence prominent chemical interaction between graphene and substrate. All in all, graphene on TiC (111) is a 
new example of strongly coupled systems. Such strong interaction is typical for Ni (111) and Co (0001) substrates. In contrast to these systems, where graphene is strained, graphene on TiC (111) is slightly corrugated with amplitude $0.5 \AA$ due to the mismatch with the substrated $\mathrm{TiC}(111)$ surface.

\section{Experimental section}

We used polished single crystals of $\mathrm{TiC}$ cut along the (111) and (755) planes (MaTecK). Clean $\mathrm{TiC}$ surfaces were prepared by heating at $1150^{\circ} \mathrm{C}$ for $20 \mathrm{~min}$ at oxygen pressure of $10^{-7}$ mbar followed by cooling down to the room temperature and subsequent fast heating (flashing) up to $1300^{\circ} \mathrm{C}$ for several times. The surface composition was well reproducible (Table S3 in the Supporting information file). It should be noted that titanium carbide has asymmetric and broad homogeneity range from about 35 to 50 at.\% $\mathrm{C}$. Potentially It can lose some amount of carbon upon surface treatment. Unfortunately, from the photoelectron diffraction effects we cannot exactly quantify surface composition. Nevertheless, we believe that the we still have stoichiometric TiC based on (i) valence band data which are very sensitive to stoichiometry [48] and (ii) the fact that we can detect easily carbon when photoelectron kinetic energy corresponds to ultimate surface sensitivity i.e. around $50 \mathrm{eV}$.

Graphene was grown by chemical vapor deposition on clean TiC (755) surfaces from propylene $\left(\mathrm{C}_{3} \mathrm{H}_{6}\right)$ at $850-1180{ }^{\circ} \mathrm{C}$ and the $\mathrm{C}_{3} \mathrm{H}_{6}$ pressure of $10^{-6}$ mbar during about 5 min followed by slow cooling to room temperature at a rate of $1-2^{\circ} \mathrm{C} / \mathrm{min}$.

The oxidation of the crystal surface was carried out at room temperature under the molecular oxygen pressure of $p \mathrm{O}_{2}=1 \cdot 10^{-6}-8 \cdot 10^{-5} \mathrm{mbar}$ for $30 \mathrm{~min}$.

$\mathrm{X}$-ray photoemission studies were performed using several facilities at Helmholtz Zentrum, Berlin (HZB). The XPS studies were performed at the RGBL beamline (HZB). High resolution Ti 2p, C 1s, and Ti 2s core level spectra were acquired at a photoelectron kinetic energy of $50 \mathrm{eV}$ to provide the ultimate surface sensitivity and at $200 \mathrm{eV}$ for composition quantification using a SPECS Phoibos 150 electron energy analyzer. The photon energies were calibrated using the second-order diffraction reflection from the plane grating.

All spectra were fitted by Gaussian/Lorentzian convolution functions using Unifit 2014 software. The background was optimized using a combination of Shirley and Tougaard functions simultaneously with the spectral fitting. Atomic fractions were calculated from the integral peak intensities obtained at a fixed kinetic energy $(200 \mathrm{eV})$ normalized by photoionization cross-sections [23] and the photon flux (see Supplementary Information for 
details). Layer thickness was estimated using the Hill equation [18] taking into account the corresponding values of inelastic mean free path calculated using the TPP 2M formula [24] and the atomic density.

The ARPES measurements of the band structure of graphene-covered TiC(755) were performed at the endstation ARPES 12 installed at the beamline UE112-PGM2 at BESSY II and equipped with a Scienta R8000 analyser. Linearly polarized light with photon energies between 50 and $90 \mathrm{eV}$ was used. Measurements we performed at room temperature. The transfer of samples between the preparation, XPS and ARPES chambers was realized with the help of a Ferrovac UHV vacuum suitcase.

\section{Acknowledgments}

We thank Helmholtz-Zentrum Berlin (HZB) for the allocation of synchrotron radiation beamtimes at the Russian-German and UE112-PGM2 beamlines. The work was financially supported by the Russian Science Foundation (project 16-42-01093).

\section{References}

[1] S.V. Didziulis, K.D. Butcher, A perspective on the properties and surface reactivities of carbides and nitrides of titanium and vanadium, Coordination Chemistry Reviews 257(1) (2013) 93-109.

[2] J.G. Chen, Carbide and Nitride Overlayers on Early Transition Metal Surfaces: Preparation, Characterization, and Reactivities, Chem. Rev. 96(4) (1996) 1477-1498.

[3] H. Itoh, T. Ichinose, C. Oshima, T. Ichinokawa, T. Aizawa, Scanning tunneling microscopy of monolayer graphite epitaxially grown on a TiC(111) surface, Surface Science Letters 254(1) (1991) L437-L442.

[4] A. Nagashima, K. Nuka, H. Itoh, T. Ichinokawa, C. Oshima, S. Otani, Electronic states of monolayer graphite formed on TiC(111) surface, Surface Science 291(1-2) (1993) 93-98.

[5] M. Terai, N. Hasegawa, M. Okusawa, S. Otani, C. Oshima, Electronic states of monolayer micrographite on TiC(111)-faceted and TiC(410) surfaces, Applied Surface Science 130 (1998) 876-882.

[6] T. Tanaka, A. Tajima, R. Moriizumi, M. Hosoda, R. Ohno, E. Rokuta, C. Oshima, S. Otani, Carbon nano-ribbons and their edge phonons, Solid State Commun. 123(1) (2002) 3336.

[7] C. Zhao, Q. Wang, H. Zhang, S. Passerini, X. Qian, Two-Dimensional Titanium Carbide/RGO Composite for High-Performance Supercapacitors, ACS applied materials \& interfaces 8(24) (2016) 15661-15667.

[8] M. Ghidiu, M.R. Lukatskaya, M.-Q. Zhao, Y. Gogotsi, M.W. Barsoum, Conductive twodimensional titanium carbide / clay/' with high volumetric capacitance, Nature 516(7529) (2014) 78-81.

[9] S.A.F. Muhammed M. Ottakam Thotiyl, Zhangquan Peng, Yuhui Chen, Zheng Liu, a.P.G. Bruce, A stable cathode for the aprotic $\mathrm{Li}-\mathrm{O}_{2}$ battery, Nature Materials (2013). [10] A.Y. Kozmenkova, E.Y. Kataev, A.I. Belova, M. Arnati, L. Gregoratti, J. VelascoVelez, A. Knop-Gericke, B. Senkovsky, D.V. Vyalikh, D.M. Itkis, Y. Shao-Horn, L.V. 
Yashina, Tuning Surface Chemistry of TiC Electrodes for Lithium-Air Batteries, Chem. Mater. 28(22) (2016) 8248-8255.

[11] C. Ruberto, B.I. Lundqvist, Nature of adsorption on TiC(111) investigated with densityfunctional calculations, Phys. Rev. B 75(23) (2007).

[12] S.V. Didziulis, K.D. Butcher, S.S. Perry, Small Cluster Models of the Surface Electronic Structure and Bonding Properties of Titanium Carbide, Vanadium Carbide, and Titanium Nitride, Inorganic Chemistry 42(24) (2003) 7766-7781.

[13] D. Jaeger, J. Patscheider, Single Crystalline Oxygen-free Titanium Nitride by XPS, Surface Science Spectra 20(1) (2013) 1-8.

[14] D. Jaeger, J. Patscheider, A complete and self-consistent evaluation of XPS spectra of TiN, Journal of Electron Spectroscopy and Related Phenomena 185(11) (2012) 523-534. [15] S.V. Didziulis, J.R. Lince, T.B. Stewart, E.A. Eklund, Photoelectron Spectroscopic Studies of the Electronic Structure and Bonding in TiC and TiN, Inorganic Chemistry 33(9) (1994) 1979-1991.

[16] G. Soto, AES, EELS and XPS characterization of Ti(C, N, O) films prepared by PLD using a Ti target in N2, CH4, O2 and CO as reactive gases, Applied Surface Science 233(1-4) (2004) 115-122.

[17] L.I. Johansson, H.I.P. Johansson, K.L. Håkansson, Surface-shifted N 1sand C 1slevels on the (100) surface of TiN and TiC, Phys. Rev. B 48(19) (1993) 14520-14523.

[18] L.I. Johansson, Surface shifts in core level energies of transition metal carbides and nitrides, in: S.T. Oyama (Ed.), The Chemistry of Transition Metal Carbides and Nitrides, Springer Netherlands, Dordrecht, 1996, pp. 455-472.

[19] K.E. Tan, M.W. Finnis, A.P. Horsfield, A.P. Sutton, Why TiC(111) is observed to be Ti terminated, Surface Science 348(1) (1996) 49-54.

[20] A. Vojvodic, C. Ruberto, B.I. Lundqvist, Atomic and molecular adsorption on transitionmetal carbide (111) surfaces from density-functional theory: a trend study of surface electronic factors, Journal of physics. Condensed matter : an Institute of Physics journal 22(37) (2010) 375504.

[21] D.W. Fischer, Molecular-Orbital Interpretation of the Soft X-Ray LII,III Emission and Absorption Spectra from Some Titanium and Vanadium Compounds, Journal of Applied Physics 41(9) (1970) 3561-3569.

[22] S. Tougaard, Energy loss in XPS: Fundamental processes and applications for quantification, non-destructive depth profiling and 3D imaging, Journal of Electron Spectroscopy and Related Phenomena 178-179 (2010) 128-153.

[23] P.M. Th, M. van Attekum, J.M. Trooster, Bulk- and surface-plasmon-loss intensities in photoelectron, Auger, and electron-energy-loss spectra of Al metal, Phys. Rev. B 18(8)

(1978) 3872-3883.

[24] M. Kurth, P.C.J. Graat, E.J. Mittemeijer, Determination of the intrinsic bulk and surface plasmon intensity of XPS spectra of magnesium, Applied Surface Science 220(1) (2003) 6078.

[25] J. Leiro, E. Minni, E. Suoninen, Study of plasmon structure in XPS spectra of silver and gold, Journal of Physics F: Metal Physics 13(1) (1983) 215.

[26] S. Hüfner, Photoelectron spectroscopy principles and applications, Springer, New York, 2013.

[27] A. Kotani, Y. Toyozawa, Photoelectron Spectra of Core Electrons in Metals with an Incomplete Shell, Journal of the Physical Society of Japan 37(4) (1974) 912-919.

[28] S. Hüfner, G.K. Wertheim, Multielectron effects in the XPS spectra of nickel, Phys. Lett. A. 51(5) (1975) 299-300. 
[29] C. Guillot, Y. Ballu, J. Paigné, J. Lecante, K.P. Jain, P. Thiry, R. Pinchaux, Y. Pétroff, L.M. Falicov, Resonant Photoemission in Nickel Metal, Phys. Rev. Lett. 39(25) (1977) 16321635.

[30] E. Grånäs, J. Knudsen, U.A. Schröder, T. Gerber, C. Busse, M.A. Arman, K. Schulte, J.N. Andersen, T. Michely, Oxygen Intercalation under Graphene on $\operatorname{Ir}(111)$ : Energetics, Kinetics, and the Role of Graphene Edges, ACS Nano 6(11) (2012) 9951-9963.

[31] S. Zaima, Y. Shibata, H. Adachi, C. Oshima, S. Otani, M. Aono, Y. Ishizawa, Atomic chemical composition and reactivity of the TiC(111) surface, Surface Science 157(2) (1985) 380-392.

[32] P.A. Khomyakov, G. Giovannetti, P.C. Rusu, G. Brocks, J. van den Brink, P.J. Kelly, First-principles study of the interaction and charge transfer between graphene and metals, Phys. Rev. B 79(19) (2009).

[33] A.B. Preobrajenski, M.L. Ng, A.S. Vinogradov, N. Mårtensson, Controlling graphene corrugation on lattice-mismatched substrates, Phys. Rev. B 78(7) (2008) 073401.

[34] E. Voloshina, R. Ovcharenko, A. Shulakov, Y. Dedkov, Theoretical description of X-ray absorption spectroscopy of the graphene-metal interfaces, The Journal of chemical physics 138(15) (2013) 154706.

[35] P.R. Wallace, The Band Theory of Graphite, Physical Review 71(9) (1947) 622-634. [36] J.W. McClure, Band Structure of Graphite and de Haas-van Alphen Effect, Physical Review 108(3) (1957) 612-618.

[37] E.L. Shirley, L.J. Terminello, A. Santoni, F.J. Himpsel, Brillouin-zone-selection effects in graphite photoelectron angular distributions, Phys. Rev. B 51(19) (1995) 13614-13622. [38] A.A. Popova, A.M. Shikin, A.G. Rybkin, D.E. Marchenko, O.Y. Vilkov, A.A.

Makarova, A.Y. Varykhalov, O. Rader, The role of the covalent interaction in the formation of the electronic structure of $\mathrm{Au}$ - and $\mathrm{Cu}$-intercalated graphene on $\mathrm{Ni}(111)$, Physics of the Solid State 53(12) (2011) 2539-2544.

[39] K. Kobayashi, Y. Souzu, s. Isshiki, M. Tsukada, Theory of STM images of monolayer graphite on transition-metal surface, Applied Surface Science 60 (1992) 443-447.

[40] A. Varykhalov, D. Marchenko, J. Sánchez-Barriga, M.R. Scholz, B. Verberck, B. Trauzettel, T.O. Wehling, C. Carbone, O. Rader, Intact Dirac Cones at Broken Sublattice Symmetry: Photoemission Study of Graphene on Ni and Co, Phys. Rev. X 2(4) (2012) 041017.

[41] D. Marchenko, A. Varykhalov, J. Sánchez-Barriga, O. Rader, C. Carbone, G. Bihlmayer, Highly spin-polarized Dirac fermions at the graphene/Co interface, Phys. Rev. B 91(23) (2015) 235431.

[42] P. Ruffieux, J. Cai, N.C. Plumb, L. Patthey, D. Prezzi, A. Ferretti, E. Molinari, X. Feng, K. Müllen, C.A. Pignedoli, R. Fasel, Electronic Structure of Atomically Precise Graphene Nanoribbons, ACS Nano 6(8) (2012) 6930-6935.

[43] B.V. Senkovskiy, A.V. Fedorov, D. Haberer, M. Farjam, K.A. Simonov, A.B. Preobrajenski, N. Mårtensson, N. Atodiresei, V. Caciuc, S. Blügel, A. Rosch, N.I. Verbitskiy, M. Hell, D.V. Evtushinsky, R. German, T. Marangoni, P.H.M. van Loosdrecht, F.R. Fischer, A. Grüneis, Semiconductor-to-Metal Transition and Quasiparticle Renormalization in Doped Graphene Nanoribbons, Advanced Electronic Materials 3(4) (2017) 1600490-n/a.

[44] I. Pletikosic, M. Kralj, P. Pervan, R. Brako, J. Coraux, T. N'Diaye A, C. Busse, T. Michely, Dirac cones and minigaps for graphene on $\operatorname{Ir}(111)$, Phys. Rev. Lett. 102(5) (2009) 056808.

[45] A. Varykhalov, J. Sánchez-Barriga, D. Marchenko, P. Hlawenka, P.S. Mandal, O. Rader, Tunable Fermi level and hedgehog spin texture in gapped graphene, 6 (2015) 7610. 
[46] S. Chen, L. Brown, M. Levendorf, W. Cai, S.-Y. Ju, J. Edgeworth, X. Li, C.W. Magnuson, A. Velamakanni, R.D. Piner, J. Kang, J. Park, R.S. Ruoff, Oxidation Resistance of Graphene-Coated $\mathrm{Cu}$ and Cu/Ni Alloy, ACS Nano 5(2) (2011) 1321-1327.

[47] B. Luo, P.R. Whelan, A. Shivayogimath, D.M.A. Mackenzie, P. Bøggild, T.J. Booth, Copper Oxidation through Nucleation Sites of Chemical Vapor Deposited Graphene, Chem. Mater. 28(11) (2016) 3789-3795.

[48] Z. Dridi, B. Bouhafs, P. Ruterana, H. Aourag, First-principles calculations of vacancy effects on structural and electronic properties of TiC x and TiN x, J. Phys.: Condens. Matter 14(43) (2002) 10237. 